\title{
BMJ Open Improving distress in dialysis (iDiD): a feasibility two-arm parallel randomised controlled trial of an online cognitive behavioural therapy intervention with and without therapist-led telephone support for psychological distress in patients undergoing haemodialysis
}

Joanna L Hudson, ${ }^{1}$ Rona Moss-Morris, ${ }^{1}$ David Game, ${ }^{2}$ Amy Carroll, ${ }^{2}$ Paul McCrone, ${ }^{3}$ Matthew Hotopf, ${ }^{4}$ Lucy Yardley, ${ }^{5}$ Joseph Chilcot ${ }^{1}$

To cite: Hudson JL, MossMorris R, Game D, et al. Improving distress in dialysis (iDiD): a feasibility two-arm parallel randomised controlled trial of an online cognitive behavioural therapy intervention with and without therapist-led telephone support for psychological distress in patients undergoing haemodialysis. BMJ Open 2016;6:e011286.

doi:10.1136/bmjopen-2016011286

- Prepublication history and additional material is available. To view please visit the journal (http://dx.doi.org/ 10.1136/bmjopen-2016011286)

Received 25 January 2016 Revised 14 March 2016 Accepted 23 March 2016 CrossMark

For numbered affiliations see end of article.

Correspondence to Dr Joseph Chilcot; joseph.chilcot@kcl.ac.uk

\section{ABSTRACT}

Introduction: Psychological distress is common in end-stage kidney disease (ESKD) and is associated with poorer health outcomes. Cognitive behavioural therapy (CBT) is recommended in UK clinical guidelines for the management of depression in people with long-term conditions. Access to skilled therapists competent in managing the competing mental and physical health demands of ESKD is limited. Online CBT treatments tailored to the needs of the ESKD population offers a pragmatic solution for underresourced services. This study examines the feasibility and acceptability of implementing a two-arm parallel randomised controlled trial of online CBT with (intervention arm) and without (control arm) therapist support to improve psychological distress in patients undergoing haemodialysis.

Methods: Patients will be screened for depression and anxiety while attending for their haemodialysis treatments. We aim to recruit 60 adult patients undergoing haemodialysis who meet criteria for mild to moderately severe symptoms of depression and/or anxiety. Patients will be randomised individually (using a 1:1 computerised sequence ratio) to either online CBT with therapist telephone support (intervention arm), or online CBT with no therapist (control arm). Outcomes include feasibility and acceptability descriptive data on rates of recruitment, randomisation, retention and treatment adherence. Self-report outcomes include measures of depression (Patient Health Questionnaire-9), anxiety (Generalised Anxiety Disorder-7), quality of life (Euro-QoL), service use (client service receipt inventory) and illness cognitions (brief illness perception questionnaire). A qualitative process evaluation will also be conducted. The statistician will be blinded to treatment allocation.

\section{Strengths and limitations of this study}

- This protocol provides a framework for the design and evaluation of an online cognitive behavioural therapy treatment for the management of comorbid distress and end-stage renal disease.

- First feasibility study to evaluate cognitive behavioural therapy using online pragmatic delivery methods in a UK National Health Service (NHS) haemodialysis setting.

- Recruitment from a single UK NHS site may hinder generalisabilty of feasibility outcomes.

- Patient treatment preferences are not accounted for. However, such designs would be associated with increased costs, a prohibitive factor in the current study, and increase the potential for confounding factors.

Ethics and dissemination: A National Health Service (NHS) research ethics committee approved the study. Data from this study will provide essential information for the design and testing of further interventions to ameliorate distress in patients undergoing dialysis. Any amendments to the protocol will be submitted to the NHS committee and study sponsor.

Trial registration number: NCT023528702; Pre-results.

\section{INTRODUCTION}

End-stage kidney disease (ESKD) is a chronic condition that permanently affects kidney 
function. ${ }^{1}$ Without renal replacement therapy (eg, dialysis or transplantation) a person's physical health would rapidly deteriorate because of the build-up of toxins and waste products in the body. ${ }^{2}$ In addition to renal replacement therapy, patients are required to attend regular clinical appointments, take multiple medications, and adhere to rigid dietary and fluid restrictions. ${ }^{3}$

Psychological distress is common in ESKD with an estimated prevalence of $39 \%$ among people in receipt of dialysis compared with a prevalence of $27 \%$ in patients with chronic kidney disease (stages $1-5) .{ }^{4}$ Comorbid psychological distress and ESKD are associated with higher rates of mortality ${ }^{5}$ and healthcare usage. ${ }^{6}$ The safety and efficacy of pharmacotherapy in managing psychological distress among people with ESKD remains unclear because of a lack of robust randomised controlled trials (RCTS). ${ }^{7}$ Although talking therapies likely offer a safer alternative to pharmacotherapy, their efficacy in the ESKD population is largely unknown. Only two small-scale, non-UK based, RCTs have examined the efficacy of cognitive behavioural therapy (CBT) relative to usual care among patients undergoing haemodialysis. ${ }^{8} 9$ Both trials found CBT was effective in reducing psychological distress. These findings are consistent with larger scale RCTs of CBT treatments for depression in people with coronary heart disease. ${ }^{10}$

The NHS has limited resources to allow the demand for CBT therapist time to be met adequately. A practical approach to address this problem is to implement a stepped-care health service delivery model. ${ }^{11}$ Within this model, individuals begin with low-intensity interventions unless their distress is deemed too severe to benefit from the type of minimal intervention offered. Providing low-intensity treatments means that there is decreased treatment burden for patients, but equally, health services can treat a larger volume of patients. If necessary, a patient is 'stepped up' to receive more intensive intervention if the initial low-intensity treatment did not improve outcomes.

Guided self-help CBT treatments are considered lowintensity interventions ${ }^{12}$ and are effective in the management of psychological distress in people with ${ }^{13}$ and without comorbid physical health conditions. ${ }^{14}$ Online/ computerised self-help resources allow better management of the informational needs of patients, and encourage active engagement with treatment by interacting with the online interface. ${ }^{15}$ Indeed, in people without physical health conditions, online/computerised guided self-help treatments have largely demonstrated equivalence with face-to-face psychological interventions in terms of their clinical effectiveness (depression and anxiety) ${ }^{16}$ and degree of adherence to treatment sessions. ${ }^{17}$

However, there are a number of factors that determine the efficacy of online and computerised self-help treatments. One moderating factor is whether support is provided by a healthcare professional. Online/computerised self-help treatments with support from healthcare professionals improves outcomes and prevents treatment dropout. ${ }^{18} 19$ The type of support provided is also important. A recent RCT explored the efficacy of online CBT with weekly technical/motivational support calls from a non-clinician for the management of depression, and compared it with usual general practitioner (GP) care. $^{20}$ Its findings confirmed that providing patients with access to online CBT with only technical support had no added benefit on depression outcomes compared with GP usual care. Access to a skilled therapist is especially important in the context of comorbid mental and physical health conditions because of the potential for treatment antagonisms, whereby the effective management of a person's mental health has the potential to dysregulate the management of physical health or vice versa. ${ }^{21}$

Given that the evidence points to the efficacy of online/computerised treatments with therapeutic support for the management of psychological distress in people with and without physical long-term condition (LTCs), it remains uncertain whether these findings apply to the management of psychological distress in UK NHS haemodialysis treatment settings. This study seeks to explore the feasibility and acceptability of implementing a two-arm parallel RCT of online CBT with (intervention arm) and without (control arm) therapist support to improve psychological distress in people receiving haemodialysis treatments within a stepped-care health service delivery framework.

\section{Background to the study}

The development of the improving distress in dialysis (iDiD) online CBT treatment involved a multidisciplinary team of health psychologists, clinical psychologists, psychiatrists, nephrologists and six patient and public involvement representatives. The preliminary content of the website was initially determined by self-help resources used to manage adjustment outcomes in LTCs implemented in previous trials by one of our authors (RMM) ${ }^{22-24}$ In addition, a literature review of the correlates of distress in dialysis was used to develop an ESKD-specific CBT treatment formulation and sevensession protocol (JL Hudson, R Moss-Morris, D Game, et al. Improving distress in dialysis (iDiD): a tailored CBT treatment for dialysis patients. Under review. 2016). In brief, our CBT formulation recognises the unique acute and chronic stressors that occur in the dialysis population including: ESKD diagnosis, surgical procedures needed for vascular access site generation, loss of independence, changes in body and self-image, uncertainty about health and future, the unseen burden of kidney disease, and chronic illness self-management challenges, specifically managing thirst and food cravings, and dealing with health professionals. CBT intervention techniques for managing these illness-specific stressors are then introduced in subsequent online treatment sessions. The content of each treatment session was first drafted on paper and reviewed by the research team. Patient representatives then provided feedback on 
the relevance and ease of understanding of the information and CBT intervention techniques described. Next, the intervention content was uploaded onto an online platform using LifeGuide software. ${ }^{25}$ The presentation and navigation through the website was tested using patient representatives and 'think-aloud' techniques. This process occurred iteratively so that comments on early sessions could be incorporated into the design of subsequent sessions.

The intervention includes a total of seven sessions. The content of each session is summarised in online supplementary table S1. For more detailed information, see our distress in dialysis CBT treatment formulation model (JL Hudson, et al. Improving distress in dialysis (iDiD): a tailored CBT treatment for dialysis patients. Under review. 2016). Patients are encouraged to complete one session per week, and each session was designed to last approximately 1 hour in duration.

\section{OBJECTIVES}

The following aims will be addressed as part of a feasibility and acceptability parallel RCT of online CBT with and without therapist support, delivered within a stepped-care framework among outpatient patients undergoing haemodialysis, with comorbid psychological distress:

- To assess the feasibility and acceptability of screening all patients who attend for haemodialysis. Patients will be screened for depression and anxiety using standardised measures presented on iPADs. The presence of psychological distress is often not identified by nonmental health-trained clinicians. ${ }^{26}$ Implementing screening questionnaires for psychological distress in medical settings can promote its detection ${ }^{27}$ and, ultimately, the provision of mental healthcare. We will quantify the number of people who agree to be screened.

- To explore rates of recruitment and retention into the trial.

- To examine willingness to be randomised to either the intervention arm (with telephone support) or control arm (no telephone support) by recording participant reasons for non-consent into the study (if disclosed).

- To explore the level of adherence to online treatment sessions and telephone support calls (intervention arm only).

- To explore the potential efficacy of an online intervention with therapist-led telephone support in reducing psychological distress when compared with website alone. This will inform the planning of a future full-scale trial to detect clinically meaningful change in outcomes of psychological distress.

- To examine if change in quality of life differs between the intervention arm and control arm.

- To provide a preliminary assessment of the costeffectiveness of the intervention.
- To examine change in ESKD illness cognitions, and whether their effect differs between the intervention and control arm.

- To qualitatively explore patient perceptions of the acceptability and usability of the website and telephone support calls, and identify areas of improvement for future interventions.

\section{METHODS}

\section{Design}

A two-arm parallel randomised controlled feasibility trial (RCT). Participants will be randomised, individually, using a 1:1 ratio computerised algorithm. A nested qualitative study will evaluate patient experience.

\section{Setting and participants}

Participants will be recruited from haemodialysis units at Guy's and St Thomas' hospital (London, UK).

Participants will be eligible for inclusion if:

- aged 18 years or over, and receive hospital haemodialysis three times weekly;

- they have mild to moderately severe depressive symptoms (based on PHQ-9 ${ }^{28}$ scores of 5-19; a self-report measure of depression) and/or presence of mild to moderately severe anxiety symptoms (based on selfreport GAD- $7^{29}$ scores of 5-14);

- they speak English sufficiently well to engage with screening tools;

- they have a basic understanding of how to use the internet and an email address.

Participants will be ineligible if

- currently receiving active treatment for depression and/or anxiety. Active treatment is defined as any current psychological treatment (talking therapies) or receipt of a new antidepressant and/or antianxiety medication. A medication is considered new if started 3 months prior to the completion of the depression and anxiety screening questionnaire;

- they have a severe mental health disorder, for example, psychosis, bipolar disorder;

- they have active suicidal thoughts, as indicated by a score of $>1$ on the depression PHQ-9 item 'Thoughts that you would be better off dead, or of hurting yourself';

- they have evidence of addiction to alcohol or drugs. A participant will be withdrawn from the study if there are safety concerns in relation to their physical or mental health

- the participant chooses to withdraw from the study

- a patient's level of psychological distress deteriorates.

\section{Flow of recruitment and participant timeline}

Participants will be identified for inclusion using webbased screening questionnaires routinely used as part of the Integrating Mental and Physical healthcare: research, training and services (IMPARTS) initiative at Guy's and St Thomas' hospital. ${ }^{30}$ Participants consenting 
to the screen will be assessed for depression and anxiety using the PHQ- $9^{31}$ and GAD-7, ${ }^{29}$ respectively. The PHQ-9 is a nine-item self-report questionnaire deemed acceptable for the identification of depression in medical care settings, including specialist settings. ${ }^{27}$ Likewise, the GAD-7, is a self-report seven-item questionnaire with evidenced criterion validity for the detection of generalised anxiety disorder. ${ }^{29}$ The PHQ-9 and GAD-7 are routinely used in UK primary care Improving Access to Psychological Therapy (IAPT) sites $^{32}$ to monitor patient outcomes. The patient will complete the web-based questionnaires either alone or with the assistance of a renal nurse/researcher. While completing the screening questionnaire, patients will be asked to give their permission (yes/no) for a member of the research team to contact them about the present study.

Results from the screening questionnaires are uploaded onto the patient's electronic medical record. Results will be checked by the nursing team/researcher for immediate risk. Risk is defined as a score of $>1$ on the depression PHQ-9 item 'Thoughts that you would be better off dead, or of hurting yourself'. If suicidal ideation is detected, then a risk assessment will be performed to determine the immediacy of referral to either liaison psychiatry or renal clinical psychology. Level of risk will be assessed in line with the IMPARTS risk assessment protocol. This includes enquiring about degree of suicidal ideation and level of hopelessness, whether active plans are present, enquiring about the patient's history of suicide attempts, recent life stressors, protective factors and degree of social support. The outcome of the risk assessment will be immediately discussed with either the renal clinical psychologist or liaison psychiatrist, and a management plan will be put into place.

Anonymised screening results will be securely emailed from the IMPARTS database to the iDiD research team on a weekly basis. A stratified stepped-care model, according to the criteria outlined in figure 1 will be applied to the anonymised data to identify potentially eligible participants for the study. The stratified steppedcare approach assigns individuals to treatments of varying intensity based on the severity of their symptoms. ${ }^{33}$ PHQ-9 scores within the range of 5-19, are considered indicative of mild to moderately severe symptoms of depression. ${ }^{31}$ Likewise, GAD-7 scores within the range of 5-14 indicate the presence of mild to moderately severe anxiety. ${ }^{29}$ Individuals with mild to moderately severe symptoms of depression and/or anxiety will be considered appropriate for treatment with the iDiD online CBT programme, and for inclusion in this study. Individuals with severe depression (PHQ-9 score $\geq 20$ ) and/or anxiety (GAD7 score $\geq 15$ ), or individuals with evidence of current suicidal ideation are considered inappropriate for $\mathrm{iDiD}$ online $\mathrm{CBT}$. These patients will be referred and managed by either the renal clinical psychology team or liaison psychiatry (when detected at the point of screen).

Individuals who meet the criteria for mild to moderately severe symptoms of depression and/or anxiety will have their data de-anonymised providing they give us consent to contact them about the study. These potential participants will be screened against the remaining inclusion/exclusion criteria during weekly referral meetings with the research and clinical team. If they remain eligible a researcher will approach the participant while they attend dialysis, to explain the study with the participant information sheet. Participants will be given a minimum of $24 \mathrm{~h}$ to establish if they would like to take part.

All patients with mild to moderately severe symptoms of depression and/or anxiety who either: (1) do not meet our remaining study inclusion criteria, (2) choose not to consent into the study, or (3) do not provide consent for us to approach them about the study, will be provided with the option of receiving usual care follow-up from the renal clinical psychology team. Usual

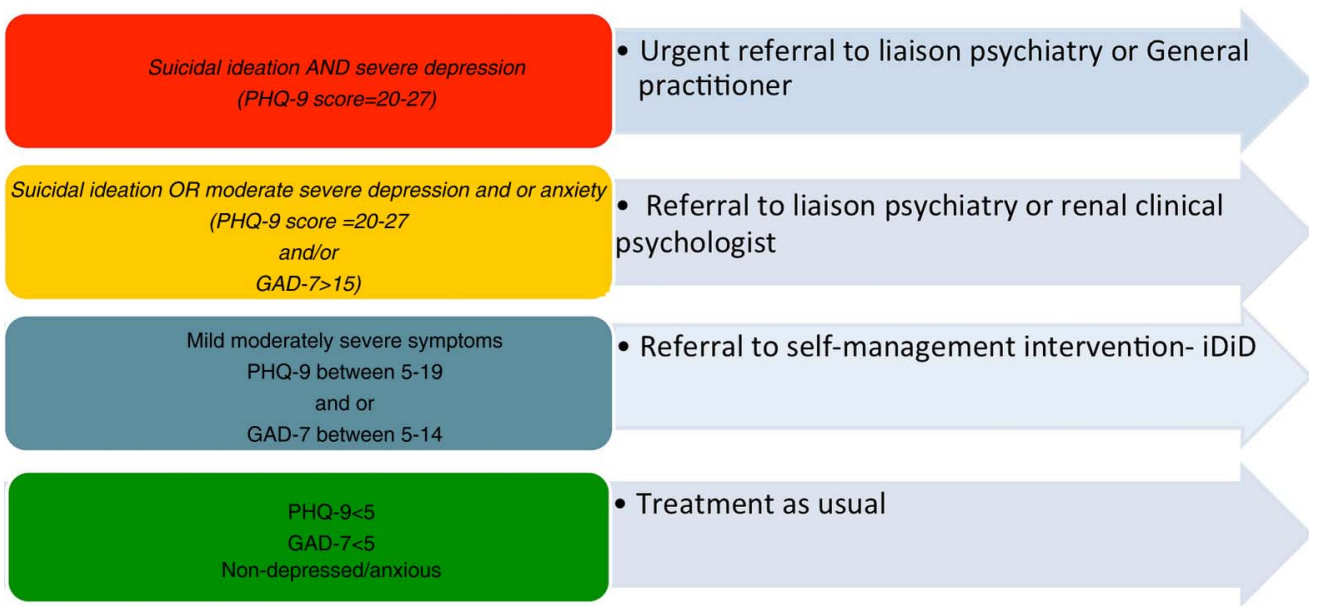

Figure 1 Stratified stepped-care referral pathway for managing psychological distress among individuals attending for haemodialysis. 
care includes a face-to-face clinical assessment followed by a tailored psychological treatment intervention or referral to an IAPT service.

As discussed above, patients who screen positive for severe symptoms of depression and/or anxiety (PHQ-9 score $\geq 20$ and/or GAD7 score $\geq 15$ ) will receive an automatic referral to the renal clinical psychology team. If on clinical assessment by the renal clinical psychologist a severely depressed and/or anxious patient is deemed appropriate for the iDiD self-help study, then they will be 'stepped down' for approach by the iDiD research team. Likewise, the research team will be informed by the renal clinical psychology team of any patients who meet the iDiD study inclusion criteria, and declare an interest in the study despite initially stating during screening that they did not want to be contacted by the study team.

Participants who consent to take part in the study will be issued with an iDiD study identification number. A researcher will attend the dialysis unit and help the patient to sign-up to the iDiD online CBT treatment using an iPAD. At sign-up, participants will be asked to enter their personal email address and select a password for use each time they logon. At the point of sign-up participants will also be asked to enter their NHS number (supplied to them by the researcher) to ensure that multiple iDiD accounts are not registered by the same participant. Participants will then receive a confirmatory email with a link to the iDiD website. After signing up, participants will complete the baseline questionnaires online. If baseline questionnaires are not completed, then participants will receive two reminder emails and an assistance-based telephone call/visit at the dialysis unit. Participants will be informed of the outcome of their randomisation process immediately after completing the online baseline questionnaire. Participants will also receive an email confirming their treatment allocation. We anticipate the participant's journey through the study will last approximately 6 months, as summarised in figure 2. We expect a period of 1 month to elapse from the point of screening to randomisation. Once participants are randomised, both groups will be able to access the iDiD website for a period of 12 weeks before being prompted to complete the follow-up questionnaires via email. The email will also advise participants that their access to the iDiD website is ending within a few weeks, and to print out any information they have found helpful from the "My tasks' tab of the website. Two reminder emails and an assistance-based telephone call/visit will occur over a period of 3 weeks if the follow-up questionnaires remain incomplete. After 20 weeks, participants will receive an email thanking them for their participation in iDiD study. Access to the $\mathrm{iDiD}$ website will no longer be available after this time. On completion of the 3-month follow-up questionnaires, a subsample of participants will be asked to complete a qualitative interview. We will follow-up participants for a period of approximately

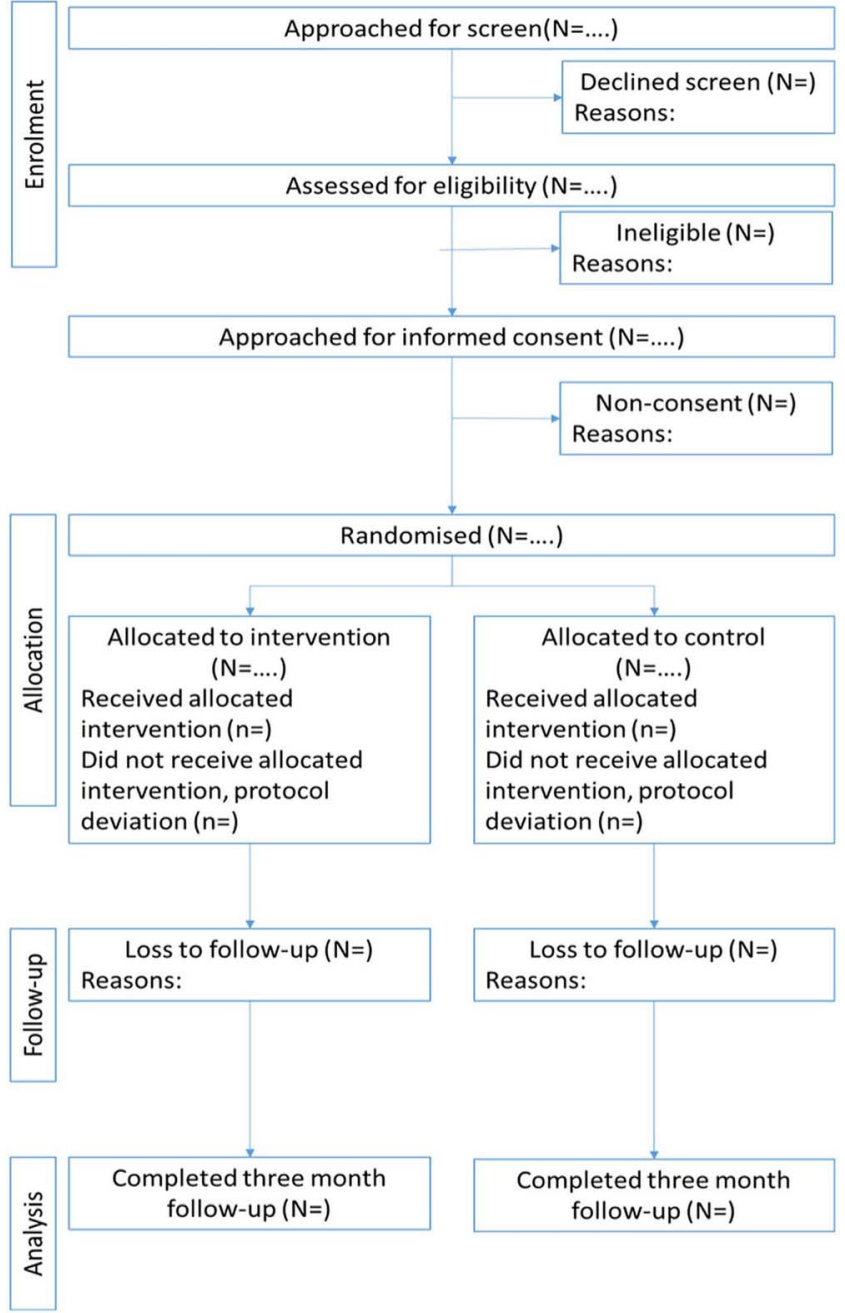

Figure 2 Flow of participants through the study.

6 months post their randomisation date, to complete the interview.

\section{Randomisation, allocation concealment and blinding}

Participants will be randomised to iDiD online CBT plus therapist-led telephone support, or iDiD online CBT-only condition using Lifeguide software (a computerised random number generator with a 1:1 ratio). Because the randomisation sequence is automated by Lifeguide in real time, the allocation sequence is concealed from researchers. All baseline questionnaires will be completed online prior to randomisation. Participants will be randomised at the individual level. The trial coordinator will also receive an automated email informing them of the outcome of the randomisation procedure to identify participants who require telephone support calls during the trial. The researcher conducting the qualitative interviews will also be unblinded at follow-up to ensure that appropriate questions are asked in relation to telephone support calls. Owing to the nature of the intervention, patients will not be blind to their treatment allocation. Follow-up outcomes will be completed independently by participants 
when prompted by email unless the participant requires assistance. The statistician will remain blinded to treatment allocation.

\section{Trial intervention}

All participants have access to the iDiD online CBT treatment (summarised in online supplementary table S1 and described in detail in our ESKD CBT formulation model) (JL Hudson, et al. Improving Distress in Dialysis (iDiD): A tailored CBT treatment for dialysis patients. Under review. 2016). Participants will be advised in the participant information sheet to logon to the website once a week. Participants will also receive weekly reminder emails to encourage engagement with the website. iPADs will be available for participants to use during their dialysis sessions.

\section{iDiD online CBT website plus therapist-led telephone support (intervention arm)}

Participants in the intervention arm of the trial will receive three $30 \mathrm{~min}$ telephone support calls at weeks 2 , 4 and 6 from JLH who has a PhD in health psychology and is a trained psychological well-being practitioner (PWP). PWPs typically work in primary care mental health teams as part of the UK Improving Access to Psychological Therapies initiative. ${ }^{32}$ PWPs deliver lowintensity CBT treatments including: cognitive restructuring, behavioural activation, problem solving, medication management, exposure therapy and sleep management. The purpose of the telephone support calls are to promote engagement with the website and to support the patient in collaboratively developing goals to work on using the resources and information available to them on the website. At the start of each telephone call, the PWP will set an agenda with the participant. The first telephone support call is scheduled for when the participant will have completed session two online. During session two the participant will have completed a self-assessment and developed their own personal model of distress. Thus, during the first call, the PWP will develop a shared understanding of the participant's source of distress, provide empathy, reinforce with the participant the relationships between thoughts, feelings and behaviours, and inform participants of the content the website which is likely most applicable to them as they continue to move forward with the website. The PWP and patient will develop a goal to work towards prior to their next telephone call.

The second telephone support call will provide an opportunity for the PWP to review with the participant their progress on their self-generated goals, work through a particular cognitive behavioural intervention technique selected by the participant, and close the session with a shared goal to work towards with the help of the website in advance of the final telephone support call. The final telephone support call will follow a similar format except the telephone session will end with a relapse prevention plan. The plan will be generated collaboratively with the patient. All telephone support calls will be audio-recorded to provide intervention fidelity checks, and for self-reflection during clinical supervision.

\section{Clinical supervision}

JLH was trained to deliver the telephone support calls using role-played sessions with feedback from RM-M. Ongoing supervision will be provided by a renal clinical psychologist (AC). Shared reflection on audiorecorded sessions will be discussed in line with the core competency framework for delivering psychological therapies in long-term conditions. ${ }^{34}$ Shared goals will be identified for the PWP to work towards over the course of the study. Supervision will also be an opportunity for case management. The PWP will discuss their proposed treatment plan in response to the first telephone support call and degree of patient progress at each supervision session. If a patient needs to be stepped-up to receive more intensive psychological treatments then this will be initiated by the research team and managed by the renal clinical psychologist.

\section{iDiD online CBT website with no telephone support (control arm)}

Participants allocated to the control arm of the study will receive their usual renal care in addition to having access to the website. Usual care for individuals with ESKD managed with haemodialysis includes attending for dialysis three times per week for up to $5 \mathrm{~h}$ at a time. Participants can be referred or self-refer into the renal clinical psychology service, or primary care mental health service, if their symptoms of depression and/or anxiety increase. We will ask participants in the 3-month follow-up questionnaire whether they have started any new treatments for mental health since starting the study.

\section{OUTCOMES}

\section{Data collection and feasibility outcomes}

Since this is a feasibility study, our primary focus is to collect data on the feasibility and acceptability of the trial design and intervention by collecting descriptive data on recruitment and retention rates and willingness to be randomised according to CONSORT trial guidelines. ${ }^{35}$ We will also examine adherence to the online intervention and telephone support calls (intervention arm only). The degree of adherence to the online intervention will be automatically recorded by the Lifeguide software. We will calculate descriptive values for the mean number of sessions completed, the number of participants who complete all sessions, the number of participants who complete $50 \%$ or more sessions and the number of participants who complete each session. Degree of adherence to the telephone call (intervention arm only) will be recorded by the PWP. The following values will be calculated: mean number of telephone 
calls completed, number of participants who complete all telephone calls, number who complete one or more telephone call, number of participants who complete telephone sessions one, two and three, respectively and mean duration of telephone calls across all three telephone support calls. In addition to a qualitative interview (described below) patients will be asked to self-report their experience of using the iDiD website at 3 months follow-up. The open-ended questions will enquire about: (1) how useful they found the iDiD website and (2) whether they found the website easy to use. Participants will also be given the option to add any further comments.

Self-reported patient outcomes will also be collected via the iDiD website at baseline and 3 months follow-up. The assessment schedule completed by patients is summarised in table 1 and described below:

- Continuous self-report measure of depression: PHQ-9 ${ }^{31}$ (described in detail above, scale range from 0 to 27, high scores indicate greater depressive symptoms).

- Continuous self-report measure of anxiety: (GAD-7) ${ }^{29}$ (described in detail above, scale range from 0 to 21, high scores indicate greater anxiety symptoms).

- The five-item EuroQoL (EQ-5D) $)^{36}$ includes a five-item measure of health status across the following domains: mobility, ability to self-care, ability to continue with activities (ie, work, social life), pain and anxiety and depression. In addition, it has a visual

\begin{tabular}{|c|c|c|c|}
\hline \multirow[b]{2}{*}{ Assessment } & \multicolumn{3}{|l|}{ Time } \\
\hline & Screening & Baseline & 3 months \\
\hline PHQ-9 & $x$ & $x$ & $x$ \\
\hline GAD-7 & $x$ & $x$ & $x$ \\
\hline EQ-5D & & $x$ & $x$ \\
\hline $\begin{array}{l}\text { Client service receipt } \\
\text { inventory }\end{array}$ & & $x$ & $x$ \\
\hline Sociodemographics & & $x$ & \\
\hline $\begin{array}{l}\text { Clinical } \\
\text { characteristics }\end{array}$ & & $x$ & \\
\hline $\begin{array}{l}\text { Biological clinical } \\
\text { outcomes }\end{array}$ & & $x$ & $x$ \\
\hline $\begin{array}{l}\text { Self-reported adverse } \\
\text { events }\end{array}$ & & & $x$ \\
\hline $\begin{array}{l}\text { Self-reported } \\
\text { treatments for } \\
\text { depression and } \\
\text { anxiety during the } \\
\text { study }\end{array}$ & & & $x$ \\
\hline $\begin{array}{l}\text { Brief illness } \\
\text { perception } \\
\text { questionnaire }\end{array}$ & & $x$ & $x$ \\
\hline Satisfaction with care & & $x$ & $x$ \\
\hline $\begin{array}{l}\text { Experience of using } \\
\text { the iDiD website }\end{array}$ & & & $x$ \\
\hline
\end{tabular}

analogue scale ranging from 0 to 100 where a person is asked to rate their overall health. The EQ-5D is recommended by NICE for use in cost-effectiveness evaluations. ${ }^{37}$

- The Client Service Receipt Inventory (CSRI) ${ }^{38}$ collects retrospective data on service use across the following five domains: (1) background and client information (ie, hospital admissions and discharge, frequency of GP visits, medications), (2) accommodation and living situation, (3) employment history, earnings and other personal resources, (4) service receipt (ie, hospital appointments, home help) and (5) receipt of informal care from caregivers. The CSRI was amended to make its content relevant to the needs of the dialysis population in collaboration with the trial health economist (PM) and renal consultant (DG).

- The brief illness perception questionnaire (BIPQ $)^{39}$ will assess participants self-reported beliefs about their ESKD. The BIPQ was developed and validated among patients with long-term conditions, including renal disease. This information will be assessed at baseline and follow-up. It will provide an indication of whether participants' beliefs about their ESKD change in response to clinical intervention.

- Satisfaction with care will be evaluated using a two-item scale that asks participants to rate their degree of satisfaction with the care they receive for their physical and mental health on a five-item Likert response scale. This information will be assessed at baseline and follow-up.

- Serious adverse events will be directly enquired about using self-report at follow-up only, according to good clinical practice guidelines. Participants will be asked whether they have experienced any adverse events since starting the study choosing from a list of five options. If participants indicate they have experienced adverse events then they will be asked for details. In addition, participants will be asked if they have experienced any adverse health effects since starting the study and encouraged to elaborate where needed.

- Treatments for depression and/or anxiety: Two brief self-report questions at follow-up will ask participants if they have received any pharmacological or psychological treatments for their depression and/or anxiety in addition to the $\mathrm{iDiD}$ website since starting the study.

\section{Sociodemographic and clinical characteristics}

Sociodemographic characteristics including: gender, age, ethnicity, home environment (marital status, housing situation, number of dependents) and level of education will be collected at baseline only via selfreport. Clinical characteristics including: dialysis vintage and treatment history for depression and anxiety will be self-reported by patients at baseline. 
Number and type of comorbidities will be extracted from notes at baseline only. The following clinical outcomes and covariates will be extracted from notes at baseline and follow-up: $\mathrm{Kt} / \mathrm{V}$ (dialysis treatment adequacy), haemoglobin, serum albumin, $\mathrm{C}$ reactive protein, serum potassium levels, interdialytic weight gain and serum phosphate levels.

\section{QUALITATIVE INTERVIEWS}

Qualitative interviews with a subgroup of participants over the phone will be conducted postintervention (3 months) by a researcher who has not been involved in their treatment. These interviews will explore whether the intervention met patient expectations, positive and negative opinions about the website, whether patients felt they gained any benefit from using the website, its personal relevance to them and its acceptability as a treatment. A minimum of 10 participants will be purposively sampled across a range of sociodemographic and clinical characteristics (eg, treatment group, age, gender, ethnicity, dialysis vintage, degree of adherence to the intervention, degree of improvements in outcomes from the intervention). Interviews will continue until data saturation occurs. The outcomes of these qualitative interviews will help to revise our theoretical understanding of distress in dialysis and update the content of the intervention accordingly, in line with current medical research guidelines for process evaluations. ${ }^{40}$ Interviews will be transcribed verbatim, and an inductive thematic analysis will be performed.

\section{SAMPLE SIZE}

The aim of this study is to explore the feasibility of implementing our trial procedures and to inform a power calculation for a future RCT. We have calculated the sample size required based on the margins of error associated with recruitment. The approximate size of the Guy's and St Thomas' dialysis population is 600 patients, in which we expect to be able to approach 400 of them. Assuming a conservative uptake rate of $50 \%$, 200 patients will be screened, with approximately $40 \%$ meeting the inclusion criteria (the estimated prevalence of depression symptoms in HD patients ${ }^{4}$ ). If we assume $50 \%$ of those eligible will consent to be randomised, a sample size of approximately 66 would allow us to estimate the true population consent rate with a $5 \%$ margin of error and a $95 \%$ confidence level.

\section{ANALYSIS PLAN}

To examine the feasibility and acceptability of our screening, recruitment, retention and randomisation process (objectives 1-3), we will quantify the flow of participants through the study using frequencies and percentages in accordance with the consort flow diagram ${ }^{35}$ shown in figure 2. We will also record and quantify reasons for non-consent, exclusion and drop-out for each stage of the study. We will examine degree of adherence to the intervention and telephone support calls (where applicable) using descriptive statistics (objective 4).

We will also perform an exploratory intention-to-treat mixed-model analysis blind to treatment group on the following self-report outcomes at 3 months follow-up: depression, anxiety and quality of life (objectives 5 and 6). Variability in these patient outcomes will help to inform a future power calculation for a full-scale trial.

Service costs will be calculated by combining service use data with appropriate unit costs. ${ }^{41}$ These will be added to the costs of the intervention which will be based on development costs and the time spent providing telephone support. Costs will be compared between the two groups, and cost-effectiveness assessed by combining the costs with the primary outcome measures and quality-adjusted life years (QALYs) in the form of incremental cost-effectiveness ratios (ICERs). Uncertainty around the ICERs will be addressed using costeffectiveness planes and acceptability curves (objective 7). We will also perform an exploratory process analysis using intention-to-treat mixed-models to establish whether illness cognitions changed in response to the online intervention and whether differences occurred between the intervention and control group (objective 8). Qualitative interviews will be transcribed verbatim and analysed using thematic analysis to allow the feasibility and acceptability of the online intervention and telephone support calls to be explored (objective 9).

\section{ETHICS}

This study has ethical approval from the NHS research ethics committee $(14 / \mathrm{LO} / 1934)$, and is sponsored by King's College London.

\section{DATA COLLECTION AND MANAGEIMENT}

The IMPARTS screening interface, developed by Teleologic Ltd, is web based and installed on the server configuration at Guy's and St Thomas' Hospitals NHS Foundation Trust. The patient logs on to the system with their unique Hospital Number. Their screening results are outputted to the documents folder of the Electronic Patient Record via the most secure WiFi network within each NHS Trust.

All quantitative outcomes are measured via online questionnaires that participants will access via $\mathrm{iDiD}$ website. The information is stored on a secure server associated with the Lifeguide programme at the University of Southampton. The website prompts the participant when data is missing. Study data can only be downloaded from the server by members of the research team who are granted password access. All data will be confidentially stored in accordance with the data protection act and King's College London data management procedures. 


\section{FORMAL COMIMITTEE}

A trial management team will meet regularly to discuss the overall running of the study including: rates of recruitment, adherence to the protocol, safety and confidentiality of patients. All serious adverse events related to the study will be reported to the study sponsor, ethics committee and Guy's and St Thomas' research and development department.

\section{DISCUSSION}

Psychological distress is common in people with ESKD. However, studies examining the efficacy of either pharmacological or psychological interventions for the management of distress in dialysis are limited. Likewise, access to psychological treatment interventions tailored to the specific psychosocial stresses associated with ESKD is problematic. An online CBT treatment designed specifically to manage distress in dialysis offers a pragmatic solution to under-resourced health services, which are advised to offer integrated mental and physical healthcare treatments.

This is the first study to examine whether it is feasible to implement an RCT of online CBT with telephone support versus online CBT without telephone support within a stepped-care framework to secondary care patients undergoing haemodialysis with comorbid distress. Indeed, it will identify unique challenges that occur in the dialysis population in the recruitment and retention of patients. Likewise, the study will be able to simultaneously examine the acceptability of this treatment to patients in terms of whether its content was relevant and useful. In addition, the utility of the online mode of delivery with or without telephone support will be examined. We anticipate that the results of this trial will substantially inform the design of a future large-scale trial powered to detect the efficacy of online CBT treatments for the management of distress in dialysis.

\section{TRIAL STATUS}

The study started recruitment in February 2015. Recruitment is until February 2016 with the last patient's follow-up in May 2016. Outcomes will be disseminated at national and international conferences, and in journal articles.

\author{
Author affiliations \\ ${ }^{1}$ Health Psychology Section, Psychology Department, Institute of Psychiatry, \\ Psychology and Neuroscience, King's College London, London, UK \\ 'Guy's and St Thomas' NHS Trust, London, UK \\ ${ }^{3}$ Health Services \& Population Research, London, UK \\ ${ }^{4}$ Department of Psychological Medicine, Institute of Psychiatry, Psychology, \\ and Neuroscience, King's College London, London, UK \\ ${ }^{5}$ Psychology Department, University of Southampton, Southampton, UK
}

Contributors All authors are involved in the design of the study. JLH, RM-M, $D G, A C, L Y$ and $J C$ are involved in intervention development. PM and JC are involved in statistical analysis plan. All authors contributed to writing the protocol.
Funding This work was funded by Guy's and St Thomas' charity (GSTT, grant number: EFT130206) and also sponsored by King's College London, Mr Keith Brennan (Keith.brennan@kcl.ac.uk).

Competing interests None declared.

Patient consent Obtained.

Ethics approval NHS ethics.

Provenance and peer review Not commissioned; externally peer reviewed.

Open Access This is an Open Access article distributed in accordance with the Creative Commons Attribution Non Commercial (CC BY-NC 4.0) license, which permits others to distribute, remix, adapt, build upon this work noncommercially, and license their derivative works on different terms, provided the original work is properly cited and the use is non-commercial. See: http:// creativecommons.org/licenses/by-nc/4.0/

\section{REFERENCES}

1. Care NK. Kidney disease: key facts and figures. East Midlands Public Health Observatory. 2010:2015.

2. National Institute for Clinical Excellence. Guidance on home compared with hospital haemodialysis for patients with end-stage renal failure. National Institute for Clinical Excellence, 2002.

3. National Institute for Health and Care Excellence. Chronic kidney disease (partial update): Early identification and management of chronic kidney disease in adults in primary and secondary care. London: National Clinical Guideline Centre, 2014.

4. Palmer S, Vecchio M, Craig JC, et al. Prevalence of depression in chronic kidney disease: systematic review and meta-analysis of observational studies. Kidney Int 2013;84:179-91.

5. Farrokhi F, Abedi N, Beyene J, et al. Association between depression and mortality in patients receiving long-term dialysis: a systematic review and meta-analysis. Am J Kidney Dis 2014;63:623-35.

6. Hedayati SS, Grambow SC, Szczech LA, et al. Physician-diagnosed depression as a correlate of hospitalizations in patients receiving long-term hemodialysis. Am J Kidney Dis. 2005;46:642-9.

7. Hedayati SS, Yalamanchili V, Finkelstein FO. A practical approach to the treatment of depression in patients with chronic kidney disease and end-stage renal disease. Kidney Int 2012;81:247-55.

8. Duarte PS, Miyazaki MC, Blay SL, et al. Cognitive-behavioral group therapy is an effective treatment for major depression in hemodialysis patients. Kidney Int 2009;76:414-21.

9. Cukor D, Ver Halen N, Asher DR, et al. Psychosocial intervention improves depression, quality of life, and fluid adherence in hemodialysis. J Am Soc Nephrol 2014;25:196-206.

10. Dickens C, Cherrington A, Adeyemi I, et al. Characteristics of psychological interventions that improve depression in people with coronary heart disease: a systematic review and meta-regression. Psychosom Med 2013;75:211-21.

11. Bower P, Gilbody S. Stepped care in psychological therapies: access, effectiveness and efficiency narrative literature review. Br J Psychiatry 2005;186:11-7.

12. National Institute for Health and Clinical Excellence. Depression in adults with a chronic physical health problem: full guideline. Retrieved 21 May 2013. http://www.nice.org.uk/guidance/cg91/ evidence/cg91-depression-with-a-chronic-physical-healthproblem-full-guideline2

13. Matcham F, Rayner L, Hutton J, et al. Self-help interventions for symptoms of depression, anxiety and psychological distress in patients with physical illnesses: a systematic review and meta-analysis. Clin Psychol Rev 2014;34:141-57.

14. Gellatly J, Bower P, Hennessy S, et al. What makes self-help interventions effective in the management of depressive symptoms? Meta-analysis and meta-regression. Psychol Med 2007;37:1217-28

15. Yardley L, Morrison LG, Andreou $P$, et al. Understanding reactions to an internet-delivered health-care intervention: accommodating user preferences for information provision. BMC Med Inform Decis Mak 2010;10:52.

16. Andersson G, Cuijpers P, Carlbring P, et al. Guided Internet-based vs. face-to-face cognitive behavior therapy for psychiatric and somatic disorders: a systematic review and meta-analysis. World Psychiatry 2014;13:288-95.

17. van Ballegooijen $W$, Cuijpers $P$, van Straten $A$, et al. Adherence to Internet-based and face-to-face cognitive behavioural therapy for depression: a meta-analysis. PLOS ONE 2014;9:e100674. 
18. Spek V, Cuijpers P, Nyklícek I, et al. Internet-based cognitive behaviour therapy for symptoms of depression and anxiety: a meta-analysis. Psychol Med 2007;37:319-28.

19. Richards D, Richardson T. Computer-based psychological treatments for depression: a systematic review and meta-analysis. Clin Psychol Rev 2012;32:329-42.

20. Gilbody S, Littlewood E, Hewitt C, et al, REEACT Team. Computerised cognitive behaviour therapy (cCBT) as treatment for depression in primary care (REEACT trial): large scale pragmatic randomised controlled trial. BMJ 2015;351:h5627.

21. Detweiler-Bedell JB, Friedman MA, Leventhal $H$, et al. Integrating co-morbid depression and chronic physical disease management: identifying and resolving failures in self-regulation. Clin Psychol Rev 2008;28:1426-46

22. Everitt HA, Moss-Morris RE, Sibelli A, et al. Management of irritable bowel syndrome in primary care: feasibility randomised controlled trial of mebeverine, methylcellulose, placebo and a patient self-management cognitive behavioural therapy website (MIBS trial). BMC Gastroenterol 2010;10:136.

23. Moss-Morris R, Dennison L, Landau S, et al. A randomized controlled trial of cognitive behavioral therapy (CBT) for adjusting to multiple sclerosis (the saMS trial): Does CBT work and for whom does it work? J Consult Clin Psychol 2013;81:251-62.

24. Moss-Morris R, Dennison L, Yardley L, et al. Protocol for the saMS trial (supportive adjustment for multiple sclerosis): a randomized controlled trial comparing cognitive behavioral therapy to supportive listening for adjustment to multiple sclerosis. BMC Neurol 2009;9:45.

25. Yardley L, Osmond A, Hare J, et al. Introduction to the LifeGuide: software facilitating the development of interactive behaviour change internet interventions. Edinburgh, UK: AISB, 2009.

26. Cepoiu M, McCusker J, Cole MG, et al. Recognition of depression by non-psychiatric physicians - a systematic literature review and meta-analysis. J Gen Intern Med 2008;23:25-36.

27. Gilbody S, Richards D, Brealey S, et al. Screening for depression in medical settings with the Patient Health Questionnaire (PHQ): a diagnostic meta-analysis. J Gen Intern Med 2007;22:1596-602.

28. Kroenke K, Spitzer RL, Williams JBW. The PHQ-9. J Gen Intern Med 2001;16:606-13.
29. Spitzer RL, Kroenke K, Williams JB, et al. A brief measure for assessing generalized anxiety disorder: the GAD-7. Arch Intern Med 2006;166:1092-7.

30. Rayner L, Matcham F, Hutton J, et al. Embedding integrated mental health assessment and management in general hospital settings: feasibility, acceptability and the prevalence of common mental disorder. Gen Hosp Psychiatry 2014;36:318-24.

31. Kroenke K, Spitzer RL, Williams JBW. The PHQ-9: validity of a brief depression severity measure. J Gen Intern Med 2001;16:606-13.

32. Layard R, Bell S, Clark D, et al. The depression report: a new deal for depression and anxiety disorders. LSE London, 2006.

33. Richards DA, Bower P, Pagel C, et al. Delivering stepped care: an analysis of implementation in routine practice. Implement Sci 2012;7:3.

34. Roth A, Pilling S. A competence framework for psychological interventions with people with persistent physical health conditions. Retrieved 29 April 2015. http://www.ucl.ac.uk/clinical-psychology/ CORE/Docs/physical-health-conditions-competences/Working\% 20with\%20physical\%20health\%20conditions\%20Background\% 20document\%20for\%20web\%2013th\%20April\%202015.pdf

35. Moher D, Hopewell S, Schulz KF, et al. CONSORT 2010 explanation and elaboration: updated guidelines for reporting paralle group randomised trials. J Clin Epidemiol 2010;63:e1-e37.

36. EuroQol Group. EuroQol - a new facility for the measurement of health-related quality of life. Health Policy 1990;16:199-208.

37. National Institute for Health and Clinical Excellence. Retrieved 27 April 2015. http://www.nice.org.uk/article/pmg9/resources// non-guidance-guide-to-the-methods-of-technology-appraisal2013-pdf

38. Beecham J, Knapp M: Costing psychiatric interventions. In: Thornicroft G. ed. London: Gaskell In Measuring Mental Health Needs, 2001.

39. Broadbent E, Donkin L, Stroh JC. Illness and treatment perceptions are associated with adherence to medications, diet, and exercise in diabetic patients. Diabetes Care 2011;34:338-40.

40. Moore GF, Audrey S, Barker M, et al. Process evaluation of complex interventions: Medical Research Council guidance, 2015.

41. Curtis L. Unit costs of health and social care 2012. Personal Social Services Research Unit, University of Kent, 2012, pp 1-272. 